\title{
Association of serum lipid peroxidation and glutathione peroxidase 4 levels with clinical outcomes and metabolic abnormalities among patients with gestational diabetes mellitus: a case-control study in the Chinese population
}

\author{
Bao Zhang ${ }^{1}$, Tingting Zhang ${ }^{1}$, Siyu Hu${ }^{1}$, Lei Sun ${ }^{1, *}$ \\ ${ }^{1}$ Department of Obstetrics and Gynecology, Shengjing Hospital of China Medical University, 110004 Shenyang, Liaoning, China \\ *Correspondence: sun11@sj-hospital.org (Lei Sun) \\ Academic Editor: Graham Pawelec \\ Submitted: 14 October 2021 Revised: 3 January 2022 Accepted: 6 January 2022 Published: 16 February 2022
}

\begin{abstract}
Purpose: This study investigated the association of serum lipid peroxidation (LPO) and glutathione peroxidase 4 (GPx4) with gestational diabetes mellitus (GDM) and metabolic abnormalities in Chinese pregnant women. Methods: The present case-control study was matched at a ratio of 1:1, and it recruited 132 pairs of participants at 24-28 gestational weeks. The serum LPO and GPx4 level were determined in each subject by enzyme-linked immunosorbent assay. The associations of LPO and GPx4 with metabolic parameters were analyzed. Thereafter, this study classified all subjects based on metabolic abnormality frequency (including body mass index, blood pressure, triglycerides, and fasting plasma glucose), and explored the association of the serum LPO and GPx4 levels in relation to metabolic abnormalities and clinical outcomes. Simultaneously, logistic regression analysis was used to estimate the odds radio (OR) and $95 \%$ confidence interval (CI) expressing the association between LPO/GPx4 and metabolic abnormalities. Results: Women with gestational diabetes mellitus (GDM) in second trimester displayed an increased LPO concentration, whereas the GPx4 concentration was decreased compared with normal subjects $(174.58 \pm 22.01 \mathrm{ng} / \mathrm{mL}$ vs. $119.54 \pm 8.93 \mathrm{ng} / \mathrm{mL}, p<0.001$ and $27.31 \pm 16.88$ vs. 33.84 $\pm 19.55 \mathrm{ng} / \mathrm{mL}, p<0.001$, respectively). In addition, the GPx4 concentration was negatively associated with the plasma fasting $2 \mathrm{~h}$ plasma glucose level (2h-PG) and percentage glycated albumin (GA\%) in the second trimester. Bivariate correlation analysis revealed that in GDM patients the serum GPx4 concentration displayed a significant linear correlation with glucose metabolism indexes, including fasting plasma glucose, glycated albumin, and $2 \mathrm{~h}-\mathrm{PG}$ (all $p<0.05$ ). By contrast, there was no relationship between the serum LPO concentration and glucose metabolism $(p>0.05)$ in GDM patients. Nevertheless, the LPO and GPx4 concentrations in the second trimester were not significantly related to the pregnancy/neonatal outcomes. Moreover, after the GDM subjects were grouped based on metabolic abnormality component, the metabolic abnormality risk was elevated with the increase in the LPO concentration (elevated diastolic blood pressure, $\mathrm{OR}=1.04, p=0.048$; and high triglycerides, $\mathrm{OR}=2.19, p<0.001)$, together with a greater incidence of multiple metabolic abnormalities. Additionally, the serum LPO concentration increased with the increased metabolic abnormality frequency $(\mathrm{OR}=1.93$, 95\% CI: $1.62-2.29, p<0.001)$. Conclusions: In women with GDM, the serum GPx4 concentration was lower, which was strongly associated with second trimester glucose metabolism among the Chinese pregnant population. According to our findings, women with GDM had an increased LPO concentration, which was strongly associated with metabolic abnormalities among the pregnant women; this might be adopted as a predictor factor for metabolic abnormalities. The results of the present study suggest that a higher lipid oxidative stress and lower lipid antioxidant associated with an increased risk of GDM.
\end{abstract}

Keywords: lipid peroxidation; glutathione peroxidase 4; lipid hydroperoxide; oxygen toxicity; gestational diabetes mellitus

\section{Introduction}

Gestational diabetes mellitus (GDM) is an aberrant pathophysiological alteration in glucose metabolism among gestational women. Throughout the pregnancy, hyperglycemia may cause oxidative stress (OS) in both the fetus and the mother, which has important effects on pregnancy as well as normal childbirth, and is associated with poor fetal outcome, including giant infant, fetal distress, and an increased incidence of congenital aplasia. Globally, the morbidity in GDM continues to increase, particularly in developing countries, including India, China, and in Africa, and it thus receives considerable attention $[1,2]$.
In a recent meta-analysis that included 79,064 pregnant women, the GDM incidence was reported to be $14.8 \%$ in mainland China [3]. Currently, the pathophysiology of GDM remains strongly debated. GDM generally results from $\beta$-cell dysfunction [4] based on chronic insulin tolerance [5] in pregnancy; in this regard, tissue insulin tolerance and $\beta$-cell damage are important components in GDM pathophysiology [6]. As the pregnancy proceeds, the insulin response stimulated by nutrients progressively increases, even though glucose tolerance deteriorates mildly, and this conforms to the progressively increased insulin resistance. For pregnant women whose glucose tolerance is 
within the normal range, their insulin sensitivity is lower, while women with GDM have an even lower insulin sensitivity [7]. Such GDM-related metabolic defects occur before type 2 diabetes mellitus (T2DM) onset. Therefore, GDM is recognized as a prediabetic state $[8,9]$.

Oxidative stress frequently occurs during pregnancy; upon the increased OS level, antioxidant defenses may also be altered [10]. OS involves DNA oxidation/impairment, protein damage, and lipid peroxidation. Antioxidants and oxidants are widely investigated in T2DM as well as the related complications [11]. However, there are few data on GDM, which shares similar pathophysiology with T2DM.Some studies on GDM report increased lipid peroxidation products and reduced activities of antioxidant enzymes [12], even though there are no consistent findings. Lipid peroxidase (LPO) are produced through radical attack on the phospholipid polyunsaturated fatty acid (PUFA) residues, which later react with redox metals and eventually produce the carcinogenic and mutagenic 4-hydroxynonenal, malondialdehyde (MDA), together with additional exocyclic DNA adducts (propano and/or etheno adducts) $[13,14]$. With regard to selenoperoxidase, glutathione peroxidase 4 (GPx4) plays an important role in the anti-peroxidant defense. Combined results regarding the enzymological mechanisms of GPx4 and LPO suggest that ferrous iron-generated alkoxyl radicals from lipid hydroperoxide derivatives initiate LPO, and their associated trace elements are normally seen in aerobic metabolism [15].

A recent study found increased oxidative stress (e.g., LPO level) and decreased antioxidative defense (e.g., glutathione peroxidase [GPx] concentration) among women suffering from late-onset GDM, which might have important clinical significance in the pregnancy course and/or pathogenesis among such women [16]. Currently, no consensus has been reached about the relationship of the serum levels of GPx4 or LPO with GDM. In relation to prior work concerning the association of LPO with GDM, the present study determined the second trimester serum GPx4 and LPO concentrations. Considering the earlier detection time, it may be of greater significance in predicting and controlling GDM. The present study focused on exploring the possible associations of GPx4 and LPO with metabolic abnormalities apart from hyperglycemia during the progress of pregnancy.

\section{Materials and methods}

\subsection{Definitions and inclusion/exclusion criteria}

At 24-48 gestational weeks, each participant received an oral glucose tolerance test (OGTT). In brief, after fasting for $10 \mathrm{~h}$ or more, the women received the 75-g OGTT. GDM was classified according to the World Health Organization (WHO) [17] endorsed International Association of Diabetes and Pregnancy Study Groups [18] (fasting plasma glucose $(\mathrm{FPG}) \geq 5.1 \mathrm{mmol} / \mathrm{L}$ or 1 -h or 2 -h plasma glucose $\mathrm{PG} \geq 10.0$ or $8.5 \mathrm{mmol} / \mathrm{L}$, respectively). Diagnosis of metabolic ab- normalities according to Chinese gestational metabolic syndrome (GMS) diagnostic criteria, includes a confirmed diagnosis of GDM, triglyceride (TG) $\geq 3.2 \mathrm{mmol} / \mathrm{L}$, and a blood pressure higher than 140/90 $\mathrm{mmHg}$ [19]. According to the number of metabolic abnormalities, patients were assigned to a normal metabolic group, one metabolic abnormality group, and two or more metabolic abnormalities group.

(a) The inclusion criteria were as follows:

(1) aged 20-40 years;

(2) planning to receive regular prenatal examinations and deliver at Shengjing Hospital;

(3) diagnosed with GDM at the 24-28 gestational weeks.

(b) Participants conforming to any one of the following criteria were excluded:

(1) no available data on the last menstrual period;

(2) with fetal growth restriction, fetal malformation, or stillbirth history, or those with $\geq 3$ abortions;

(3) receiving assisted reproductive technology;

(4) with surgical or medical disorders;

(5) hepatitis B virus, human immunodeficiency virus, or syphilis carrier;

(6) diagnosed with gestational complications in addition to GDM;

(7) the use of insulin therapy or hypoglycemic drugs;

(8) with autoimmune disease, thyroid disease, tumors or hematopathy among others.

\subsection{Normal glucose tolerance group}

The NGT gestational women (NGTGW) did not receive topical or systemic medication, and had no underlying systemic disease. The NGTGW were recruited during their second trimester at the Out-patients Clinic, Department of Obstetrics and Gynecology, Shengjing Hospital of China Medical University between January 2019 and July 2021. During this period, we identified 677 gestational women who received periodic systematic examinations at the outpatient department and were undergoing comprehensive routine inpatient prenatal care, all of whom had no abnormality in physical or OGTT examinations, and had similar exclusion criteria (as the above-mentioned in GDM) matched with those in the GDM group. Informed written consent was obtained from all 649 NGTGW before participating in this study. Fig. 1 displays the participant screening and recruitment procedure.

\subsection{Selected study participants and physical measurements}

This study adopted the propensity score matching (PSM) approach to reduce bias in case control selection and to reduce the candidate clinical confounding factors at the matching ratio of 1:1 [20]. Eventually, 132 eligible NGT cases were collected based on PSM, and additional analyses were performed to match with the GDM group. Each participant and the corresponding clinical measure- 

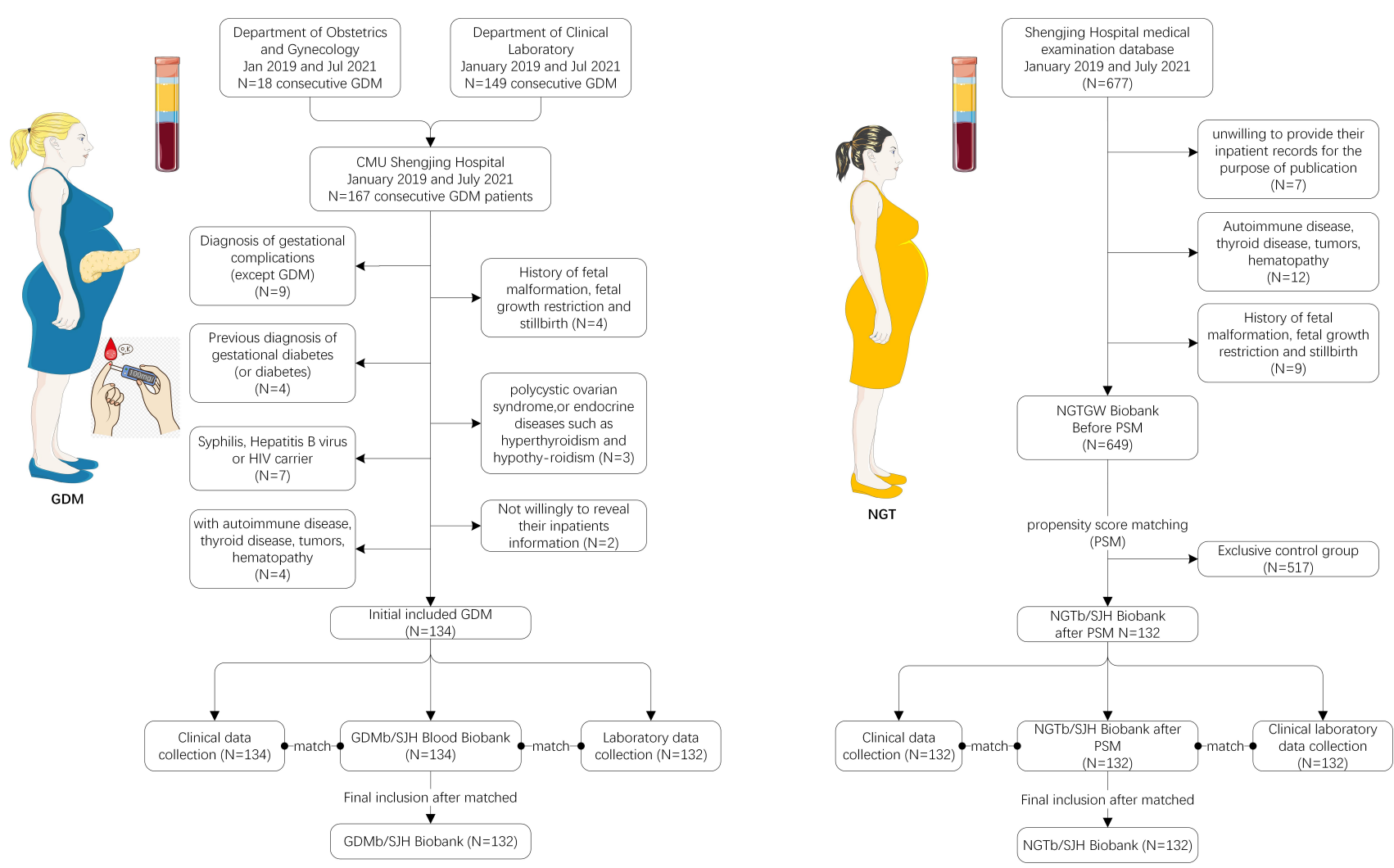

Fig. 1. Flowchart displaying the patient and blood sample screening procedures of Shengjing Hospital of blood samples for the Gestational Diabetes Mellitus Blood sample from the Shengjing Hospital Biobank (GDMb/SJH) and for the Normal Glucose Tolerance gestational women Blood sample from the Shengjing Hospital Biobank (NGTb/SJH).

ments were previously presented [21]. In total, 132 pairs of GDM cases and controls in their second trimester were recruited based on age (years), gestational week (weeks), and pregestational body mass index $\left(\mathrm{BMI}, \mathrm{kg} / \mathrm{m}^{2}\right)$. With regard to the GDM cases in their second trimester, no significant difference in age or pregestational BMI was observed in NGT subjects (both $p>0.05$ ).

\subsection{Study design}

Finally, the present case-control study recruited 264 pregnant women (132 GDM and 132 normal glucose tolerance (NGT) who were in their second trimester at the out-patients Clinic, Department of Obstetrics and Gynecology, Shengjing Hospital of China Medical University in Shenyang, China, between January 2019 and July 2021. The majority (approximately $80 \%$ ) of pregnant women living in this region (latitude $123^{\circ} \mathrm{E}$ and $41^{\circ} \mathrm{N}$ ) attend the Shengjing Hospital for antenatal care. Pregnant women who received periodic systematic examination at the outpatient department of Shengjing Hospital and GDM patients whose glucose levels were under good control were enrolled.

\subsection{GDM and NGTGW Biobank establishment}

After excluding the unqualified participants, we eliminated another 25 participants because they were unwill- ing to provide their inpatient records for the purpose of publication. We included clinical information and blood samples, respectively (Fig. 1). Finally, blood was sampled from 132 eligible GDM patients for further analysis of the Biobank that was registered as the Gestational Diabetes Mellitus Blood sample from the Shengjing Hospital Biobank (GDMb/SJH). At the same time, 132 blood was sampled from 649 NGTGW, and registered as the Normal Glucose Tolerance gestational women Blood sample from the Shengjing Hospital Biobank (NGTb/SJH). After admission, each eligible participant received an intravenous puncture for blood collection into $5.0 \mathrm{~mL}$ silica/gel plastic tubes (SST BD Vacutainer ${ }^{\circledR}$ gold) and $5.0 \mathrm{~mL}$ anticoagulant EDTA plastic tubes (BD Vacutainer ${ }^{\circledR}$ lavender). Additionally, the plasma was extracted from the sampled blood by centrifugation, which was stored at $-80^{\circ} \mathrm{C}$ before use (up to 1 year). Furthermore, peripheral blood mononuclear cells were collected from each eligible GDM patient by Ficollsodium diatrizoate density gradient centrifugation, as performed previously [22].

\subsection{Serum LPO and GPx4 measurement}

Serum LPO and GPx4 levels were determined using an enzyme-linked immunosorbent assay kit (Enzymelinked Biotechnology Co., Ltd. Shanghai, China) according to the manufacturer's instructions. This assay 
was highly sensitive to LPO (ml856631), and the range detectable concentration was $0.1-32.0 \mathrm{ng} / \mathrm{mL}$ for human LPO. In addition, this assay was also highly sensitive for GPx4 (ml060706), and the detectable concentration range was $1.0-200.0 \mathrm{ng} / \mathrm{mL}$ for human GPx4. The coefficients of variation $(\mathrm{CV})$ in the inter-assay and intra-assay were $<10 \%$ and $8 \%$, respectively.

\subsection{Clinical measurements and neonatal outcomes}

All participates (132 GDMs and 132 NGTs) were in their third trimester and planned to attend regular visits for prenatal examinations and delivery at the In-patients Clinic, Department of Obstetrics and Gynecology of Shengjing Hospital. Their clinical measurements (In-patient Clinic) in the third trimester were collected, including blood routine test, hepatic panel, lipid panel, blood coagulation function, renal function, and serum electrolyte, and the selective solubilization approach are summarized in Supplementary File 1. Blood tests were completed by the Department of Laboratory Medicine of Shengjing Hospital of China Medical University. After extracting the related clinical data, we constructed the related blood database, followed by implementation of laboratory tests. We also collected maternal clinical information, including delivery mode and gestational weeks at birth. In addition, we extracted neonatal outcomes from birth records, which included body length (cm) and birth weight (g).

\subsection{Statistical analysis}

We applied the PSM approach in this study to reduce the selection bias of case-controls and to reduce the possible clinical confounding factors at the matching ratio of $1: 1$. In addition, the model was evaluated by the HosmerLemeshow degree of fitting for the C-statistic test and logistic regression. Prior to PSM, we compared continuous variables in patient clinical features with controls by the Student's $t$-test, and compared categorical variables using the chi-squared test. Following PSM, we inspected the variable normality visually. The Mann-Whitney U-test was conducted for comparing continuous variables, whereas differences among groups were compared by the Kruskal-Wallis $\mathrm{H}$ test. The chi-squared test was conducted to compare categorical variables. In addition, the associations of GPx4 and LPO with clinical parameters were evaluated by multiple linear regression and Spearman's correlation analyses. This study adjusted confounders to conduct multiple regression analysis, including age, smoking status, blood pressure (BP), and BMI before pregnancy. Related thresholds were determined by logistic models, receiver operating characteristic (ROC) curves, and area under the ROC curve. Subsequently, the lower and upper strata of the LPO/GPx 4 concentrations were determined accordingly. This study compared the LPO/GPx4 concentrations and the pregnancy outcomes of both groups (e.g., lower strata of LPO as the reference group and upper strata of LPO as the case group). Thereafter, this study divided the subjects based on metabolic abnormality numbers (which included blood lipids, PG, and BP) as the one metabolic abnormality, $\geq 2$ metabolic abnormalities, and normal metabolic groups. Finally, the odds ratios (ORs) together with 95\% confidence intervals (CIs) were estimated by multivariate logistic regression to predict the relationship of LPO with metabolic abnormalities among the gestational women. $p<0.05$ was considered to indicate statistical significance. SPSS version 25.0 (IBM Inc., Chicago, IL, USA) and GraphPad Prism version 8.0 (GraphPad Software Inc., La Jolla, CA, USA).

\section{Results}

\subsection{Comparison of baseline clinical characteristics between the two groups}

Table 1 displays the clinical features of the 649 NGT subjects and 132 GDM cases. Among them, BMI, age, ethnicity, smokers, and hypertension showed statistical significance between the two groups prior to PSM to reduce the selection bias and possible clinical confounding factors. We subsequently compared the 132 pairs of GDM and controls using PSM by logistic regression. $p=0.524$ and $p=0.781$ were obtained upon Hosmer-Lemeshow goodness of fit test and C-statistic test, respectively. Following PSM, we excluded those heterogeneities in clinical features between the two groups. Clinical features of the GDM cases and NGT participants is elaborated in Table 1.

\subsection{Comparison of LPO and GPX4 levels between the GDM and NGT groups}

According to the WHO guidelines released in 2013, all subjects were assigned to the NGT or the GDM group. Fig. 2A exhibits the serum LPO concentrations of both groups. The GDM group displayed a markedly increased LPO concentration relative to the NGT group (174.58 \pm $22.01 \mathrm{ng} / \mathrm{mL}$ vs. $119.54 \pm 8.93 \mathrm{ng} / \mathrm{mL}, p<0.001)$. Вy contrast, the GDM group had a markedly decreased GPx4 concentration relative to the NGT group $(27.31 \pm 16.88$ vs. $33.84 \pm 19.55 \mathrm{ng} / \mathrm{mL} ; p<0.001$, Fig. 2B).

\subsection{Subgroup analysis of the comparison of the LPO and GPX4 levels within GDM}

Fig. 2C-F present subgroup analyses of the GDM patients. The serum LPO concentrations were not statistically significantly different between a family history of hypertension and normotension (Fig. 2C). The GPx4 level in the GDM without hypertension group was significantly elevated compared with the GDM with a family history of hypertension group $(49.50 \pm 52.05$ vs. $25.12 \pm 3.96 \mathrm{ng} / \mathrm{mL}, p$ $=0.041$; Fig. 2D). The serum LPO concentrations were not statistically significantly different between the primipara and pluripara within the GDM patients $(140.95 \pm 62.06$ vs. $129.19 \pm 33.31 \mathrm{ng} / \mathrm{mL}, p=0.451$; Fig. $2 \mathrm{E})$, as was the case for the GPx4 level $(26.32 \pm 13.47$ vs. $29.63 \pm$ $22.67 \mathrm{ng} / \mathrm{mL}, p=0.097$; Fig. $2 \mathrm{~F}$ ). Additionally, no signif- 
Table 1. Clinical characteristics in GDM patients and control subjects before and after PSM.

\begin{tabular}{|c|c|c|c|c|c|c|}
\hline & \multicolumn{3}{|c|}{ Full cohorts } & \multicolumn{3}{|c|}{ After PSM } \\
\hline & NGTWG & GDM & $p$ value & NGTb & GDMb & $p$ value \\
\hline & 649 & 132 & & 132 & 132 & \\
\hline Age (Years) & $29.4 \pm 4.9$ & $30.8 \pm 4.0$ & 0.213 & $31.2 \pm 6.5$ & $30.8 \pm 4.0$ & 0.637 \\
\hline Ethnicity & & & 1.000 & & & 0.871 \\
\hline Han & $528(81.4)$ & $108(81.8)$ & & $110(83.3)$ & $108(81.8)$ & \\
\hline Others & $121(18.6)$ & $24(18.2)$ & & $22(16.7)$ & $24(18.2)$ & \\
\hline Pre-pregnancy BMI $\left(\mathrm{kg} / \mathrm{m}^{2}\right)$ & $23.8 \pm 4.2$ & $24.4 \pm 4.2$ & 0.170 & $25.1 \pm 2.2$ & $24.4 \pm 4.2$ & 0.454 \\
\hline Family history of hypertension & $17(2.6)$ & $4(3.0)$ & 0.768 & $2(1.5)$ & $4(3.0)$ & 0.684 \\
\hline Family history of DM & $13(2.0)$ & $6(4.5)$ & 0.113 & $8(6.0)$ & $6(4.5)$ & 0.785 \\
\hline Smokers & $10(1.5)$ & $3(2.2)$ & 0.469 & $4(3.0)$ & $3(2.2)$ & 1.000 \\
\hline $\mathrm{SBP}(\mathrm{mmHg})$ & $106.4 \pm 13.0$ & $111.9 \pm 13.4$ & 0.006 & $108.1 \pm 11.6$ & $111.9 \pm 13.4$ & 0.184 \\
\hline DBP (mmHg) & $67.1 \pm 8.2$ & $71.9 \pm 9.4$ & 0.048 & $70.4 \pm 8.0$ & $71.9 \pm 9.4$ & 0.241 \\
\hline HR (bmp) & $78.3 \pm 14.1$ & $81.5 \pm 12.8$ & 0.052 & $79.5 \pm 10.8$ & $81.5 \pm 12.8$ & 0.665 \\
\hline OGTT time (week) & $24.6 \pm 1.6$ & $24.3 \pm 1.5$ & 0.525 & $24.8 \pm 1.7$ & $24.3 \pm 1.5$ & 0.693 \\
\hline FFG (mmol/L) & $4.7 \pm 0.8$ & $5.6 \pm 1.5$ & $<0.001$ & $4.5 \pm 0.7$ & $5.6 \pm 1.5$ & $<0.001$ \\
\hline $1 \mathrm{~h} \mathrm{PG}(\mathrm{mmol} / \mathrm{L})$ & $9.6 \pm 1.6$ & $11.4 \pm 2.9$ & $<0.001$ & $9.4 \pm 1.4$ & $11.4 \pm 2.9$ & 0.025 \\
\hline 2 h PG (mmol/L) & $8.3 \pm 1.6$ & $9.4 \pm 1.9$ & 0.002 & $8.7 \pm 0.9$ & $9.4 \pm 1.9$ & 0.042 \\
\hline Gravidity & & & 0.036 & & & 0.620 \\
\hline $1, \mathrm{n}(\%)$ & $341(52.5)$ & $56(42.4)$ & & $61(46.2)$ & $56(42.4)$ & \\
\hline $2, \mathrm{n}(\%)$ & $175(27.0)$ & $37(28.0)$ & & $40(30.3)$ & $37(28.0)$ & \\
\hline $3, \mathrm{n}(\%)$ & $133(20.5)$ & $39(29.6)$ & & $31(23.5)$ & $39(29.6)$ & \\
\hline
\end{tabular}

Note, values are mean $\pm \mathrm{SD}$ or $\mathrm{n}(\%)$.

Table 2. Diagnostic value of LPO and GPx4 and their combinations for GDM patients.

\begin{tabular}{lcccccc}
\hline & AUC & $95 \%$ CI & $p$ value & Cut-off & Sensitivity (\%) & Specificity (\%) \\
\hline LPO $(\mathrm{ng} / \mathrm{mL})$ & 0.623 & $0.539-0.707$ & 0.008 & 160.54 & 0.437 & 0.813 \\
GPx4 $(\mathrm{ng} / \mathrm{mL})$ & 0.607 & $0.520-0.694$ & 0.044 & 24.81 & 0.415 & 0.770 \\
LPO+GPx4 $(\mathrm{ng} / \mathrm{mL})$ & 0.643 & $0.560-0.727$ & 0.042 & & 0.495 & 0.780 \\
\hline
\end{tabular}

icant difference was observed in the serum LPO level between those aged $<35$ years and those $\geq 35$ years $(141.02$ \pm 62.36 vs. $129.60 \pm 32.59 \mathrm{ng} / \mathrm{mL} ; p=0.364$; Fig. $2 \mathrm{G})$ nor for the GPx4 concentration $(26.46 \pm 13.55$ vs. $29.36 \pm$ $23.02 \mathrm{ng} / \mathrm{mL}, p=0.272$; Fig. $2 \mathrm{H}$ ). To determine the predictive value of LPO and PGx4 for GDM, we performed ROC curve analysis. The area under the ROC curve was 0.623 (95\% CI: $0.54-0.71, p=0.008)$ for LPO and 0.607 (95\% CI: $0.52-0.69, p=0.044$ ) for GPx4 (Table 2). Therefore, LPO and GPx4 exhibited an acceptable capacity to distinguish the GDM patients from the general population (Fig. 3).

\subsection{Relationship between serum GPx4 and glucose metabolism}

As presented in Table 3, patient features and anthropometric variables were analyzed according to their serum GPx4 and LPO concentrations (classified as four quartiles). As revealed by the Kruskal-Wallis H test, as the GPX4 concentration was elevated, differences in the percentage glycated albumin (GA\%), FPG, and 2h-PG were significant between the different groups, and vice visa $(p=0.004, p$
$=0.028$, and $p=0.010$, respectively) (Fig. 4). By contrast, the Kruskal-Wallis $\mathrm{H}$ test analysis showed that with the rise in LPO, there were no significant differences in glucose metabolism-related indicators within the GDM group (Table 2). Using bivariate correlation, this study analyzed the association of the GPx4 concentration with glucose metabolic parameters of the GDM group. The serum GPx4 level was significantly negatively correlated with the FPG $(\mathrm{r}=-0.237, p=0.004), 2 \mathrm{~h}-\mathrm{PG}(\mathrm{r}=-0.258, p=0.040)$, and $\mathrm{GA} \%(\mathrm{r}=-0.395, p<0.001)$. In addition, as revealed by partial correlation after adjusting for gestational week and age, GPx4 displayed a positive correlation with the FPG (r $=-0.282, p=0.020), 2 \mathrm{~h}-\mathrm{PG}(\mathrm{r}=-0.302, p=0.006)$, and $\mathrm{GA} \%(\mathrm{r}=-0.343, p<0.001)$.

\subsection{LPO and GPX4 associations with clinical outcomes in $G D M$}

Table 3 presents the clinical outcome features for each subject. Using LPO $=160.54 \mathrm{ng} / \mathrm{mL}$ in the second trimester as the threshold, this study classified GDM cases into LPO upper and lower strata groups. We found that GDM pa- 
A

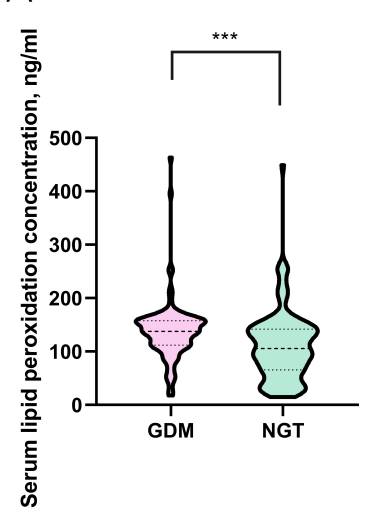

B

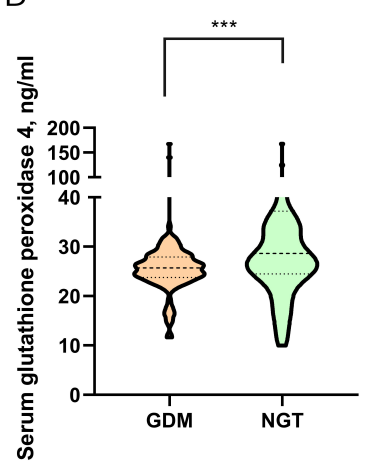

C

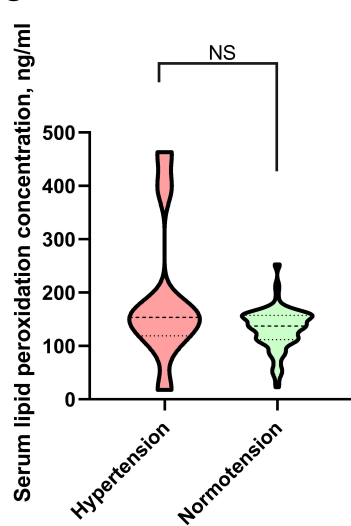

D

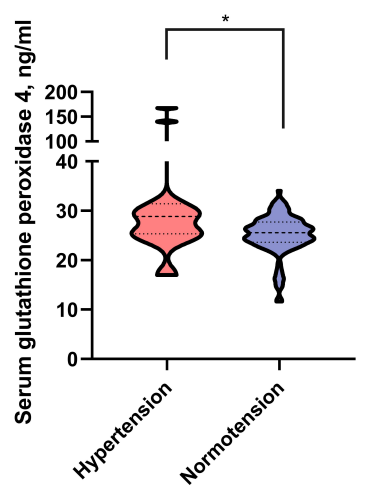

$\mathrm{E}$

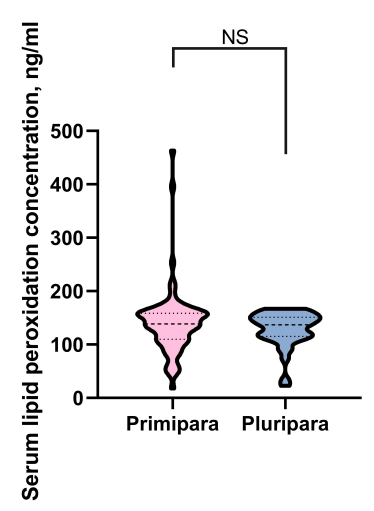

$\mathrm{F}$

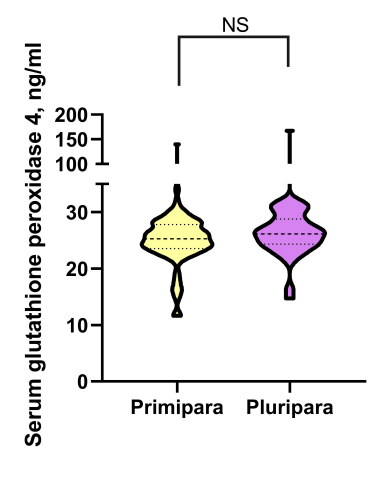

G

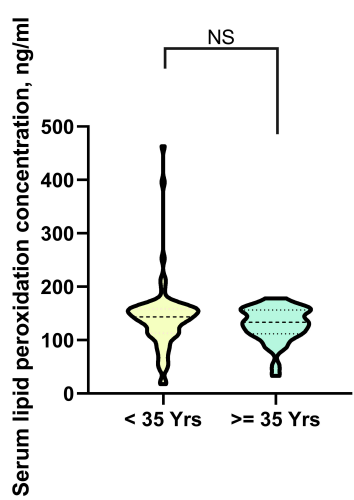

$\mathrm{H}$

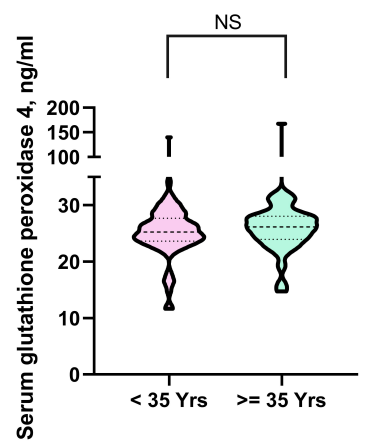

Fig. 2. The LPO (A) and GPx4 (B) concentrations of the GDM and NGT groups at 24-28 gestational weeks. Subgroup analysis between family history of hypertension and normotension (C,D), between the primipara and pluripara (E,F), and between those aged $<35$ years and those $\geq 35$ years $(\mathrm{G}, \mathrm{H})$ of serum LPO and GPx4 concentrations, respectively.

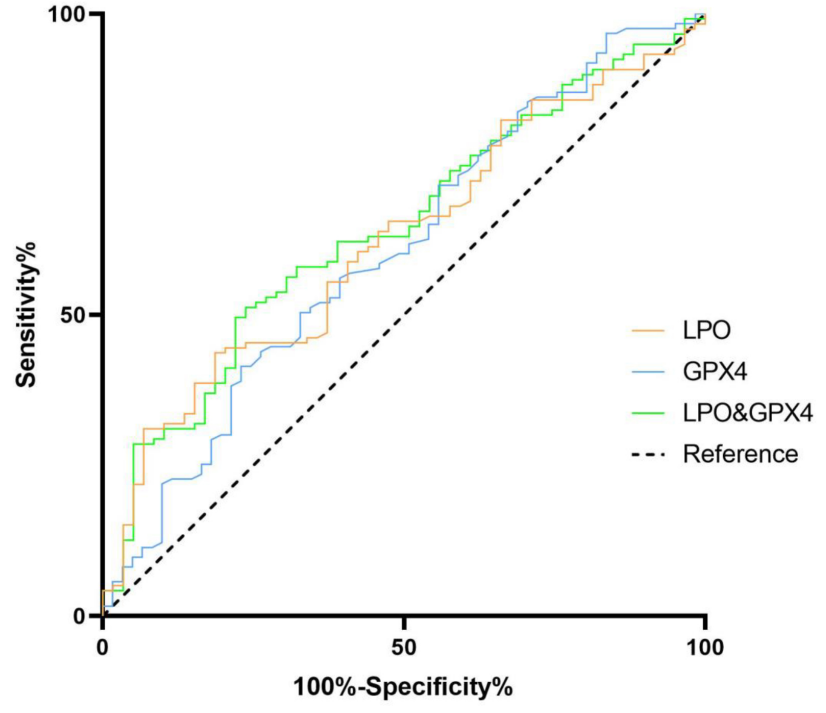

Fig. 3. ROC analysis of the LPO and GPx4 levels for the evaluation of the GDM population. The vertical axis represents the sensitivity and the horizontal axis represents the 1-specificity. tients in the second trimester with a high LPO level were more likely in the third trimester to have higher levels of total bilirubin, direct bilirubin, unconjugated bilirubin, and serum creatinine (all $p<0.05$ ). When using GPx $4=24.81$ $\mathrm{ng} / \mathrm{mL}$ as the cut-off point in the second trimester, the GDM patients were assigned to the GPx4 lower strata and GPx4 upper strata groups. We also found that GDM patients in the second trimester with a high LPO level were more likely in the third trimester to have lower TG levels $(p=0.036)$. Differences in pregnancy and neonatal outcomes were not significant between the two groups $(p>0.05$; Table 3). Furthermore, by bivariate correlation, this study analyzed the association of the LPO/GPx4 concentrations with clinical features among GDM cases, which are summarized in Fig. 4.

\subsection{Relationship between LPO and metabolic abnormalities}

In addition, multivariate logistic regression was conducted. Supplementary Table 1 presents the associations of metabolic syndrome components with the upper stratum of the LPO and GPx4 levels when compared with the 
Table 3. Anthropometric parameters and glucose metabolism variables of GDM patients at different LPO and GPx4 levels.

\begin{tabular}{|c|c|c|c|c|c|}
\hline \multirow{2}{*}{ LPO } & Quartile1 & Quartile2 & Quartile3 & Quartile4 & \multirow{2}{*}{$p$ value } \\
\hline & \multicolumn{4}{|c|}{$(<111.79 \mathrm{ng} / \mathrm{mL})(111.79-137.91 \mathrm{ng} / \mathrm{mL})(137.92-157.76 \mathrm{ng} / \mathrm{mL})(>157.76 \mathrm{ng} / \mathrm{mL})$} & \\
\hline $\mathrm{n}$ & 33 & 33 & 33 & 33 & \\
\hline Age, Years & $33.6 \pm 0.9$ & $33.4 \pm 0.8$ & $32.1 \pm 0.7$ & $33.9 \pm 1.0$ & 0.540 \\
\hline $\mathrm{FPG}, \mathrm{mmol} / \mathrm{L}$ & $5.2 \pm 0.1$ & $5.7 \pm 0.3$ & $5.3 \pm 0.1$ & $5.5 \pm 0.1$ & 0.404 \\
\hline 1h-PG, mmol/L & $10.7 \pm 0.3$ & $10.8 \pm 0.9$ & $10.8 \pm 0.4$ & $12.3 \pm 1.7$ & 0.837 \\
\hline 2h-PG, mmol/L & $9.3 \pm 0.3$ & $9.0 \pm 0.9$ & $8.4 \pm 0.4$ & $9.2 \pm 0.4$ & 0.739 \\
\hline FINS, mU/L & $13.3 \pm 1.2$ & $12.3 \pm 1.7$ & $15.6 \pm 3.5$ & $35.0 \pm 13.9$ & 0.687 \\
\hline Glycosylated hemoglobin (HbA1c), \% & $5.6 \pm 0.1$ & $5.6 \pm 0.2$ & $5.8 \pm 0.2$ & $5.6 \pm 0.1$ & 0.270 \\
\hline GA, \% & $12.1 \pm 2.9$ & $10.6 \pm 2.4$ & $13.3 \pm 3.8$ & $10.8 \pm 4.1$ & 0.459 \\
\hline \multirow{2}{*}{ GPx4 } & Quartile1 & Quartile2 & Quartile3 & Quartile4 & \\
\hline & \multicolumn{5}{|c|}{$(<23.869 \mathrm{ng} / \mathrm{mL})(23.869-25.898 \mathrm{ng} / \mathrm{mL})(25.898-27.890 \mathrm{ng} / \mathrm{mL})(>27.890 \mathrm{ng} / \mathrm{mL}) p^{p \text { value }}$} \\
\hline $\mathrm{n}$ & 33 & 33 & 33 & 33 & \\
\hline Age, Years & $33.2 \pm 0.8$ & $33.1 \pm 1.0$ & $33.1 \pm 0.9$ & $33.6 \pm 0.9$ & 0.948 \\
\hline $\mathrm{FPG}, \mathrm{mmol} / \mathrm{L}$ & $5.9 \pm 0.9$ & $5.4 \pm 0.4$ & $5.3 \pm 0.7$ & $5.1 \pm 0.4$ & 0.028 \\
\hline 1h-PG, mmol/L & $12.5 \pm 3.1$ & $11.3 \pm 1.4$ & $10.6 \pm 1.6$ & $10.5 \pm 1.1$ & 0.409 \\
\hline 2h-PG, mmol/L & $10.1 \pm 2.2$ & $10.0 \pm 1.4$ & $8.5 \pm 1.2$ & $8.3 \pm 1.4$ & 0.010 \\
\hline FINS, mU/L & $11.7 \pm 5.4$ & $19.7 \pm 12.2$ & $13.1 \pm 6.8$ & $18.6 \pm 15.7$ & 0.229 \\
\hline Glycosylated hemoglobin (HbA1c), \% & $5.4 \pm 0.5$ & $5.7 \pm 0.8$ & $5.5 \pm 0.3$ & $5.8 \pm 0.8$ & 0.233 \\
\hline GA, \% & $15.9 \pm 5.3$ & $13.0 \pm 1.2$ & $12.1 \pm 1.0$ & $9.1 \pm 3.9$ & 0.004 \\
\hline
\end{tabular}

lower stratum, according to Chinese GMS diagnostic criteria. After the GDM subjects were grouped based on the metabolic abnormality component, the metabolic abnormality risk was found to be elevated with increased LPO concentration (elevated DBP, OR $=1.04, p=0.048$; and high TGs, OR $=2.19, p<0.001$, respectively), together with a greater incidence of multiple metabolic abnormalities. According to Fig. 5, the serum LPO concentrations were greatly elevated among subjects displaying metabolic abnormalities. Subjects displaying multiple metabolic abnormalities had the highest LPO concentrations. When 'no metabolic abnormality' was utilized as the control, the presence of metabolic abnormalities in the one and multiple metabolic abnormalities groups were associated with LPO (Fig. 6).

\section{Discussion}

The present study investigated the GDM-related risk factors. The results showed that pregnant women who displayed higher LPO concentrations and lower GPx4 concentrations during the 24-28 gestational weeks were at an increased risk for GDM. This study focused on investigating the relationships of the GPX4 and LPO concentrations during the second trimester with blood glucose metabolism and metabolic abnormalities, as well as pregnancy outcomes among the GDM cases.

The studies to date have mostly focused on increased lipid oxidative stress or reduced lipid antioxidant defense, particularly in T2DM patients. For example, Bandeira et al. [23] suggested that lipid peroxidation was markedly increased in the diabetes mellitus (DM) group in compar- ison with the prediabetic groups and the normotensive and hypertension controls. Based on these findings, lipid peroxidation is related to DM, whether or not there is hypertension. As reported by some studies conducted among T2DM patients, the markers of oxidative damage increased, whereas those of antioxidant defenses decreased, in particular for the T2DM-related complications [24,25].

In the third trimester, insulin resistance that is increased physiologically may lead to elevated circulating lipids (including free fatty acids [FFAs], total cholesterol, TG, low-density lipoprotein) [10,26]. At the same time, the up-regulation of lipid OS markers is detected among cases who display various pregnancy-complicating conditions [27]. Numerous studies compared the lipid OS markers between non-pregnant and normal pregnant women [28,29]. GDM results in a higher risk for macrosomia, together with increased perinatal mortality and morbidity among fetuses, and predicts a greater risk for T2DM among the mothers [30]. In GDM, multiple metabolic defects occur before T2DM develops, therefore, GDM is recognized as a prediabetic state [8]. The pathophysiology of GDM remains strongly debated. Consequently, it is of great significance to develop an efficient approach to predict and prevent GDM. The relationships of T2DM (which shares similar clinical features with GDM) and T2DM-related complications with OS have been extensively investigated. Some studies demonstrated the imbalance between lipid antioxidation and pro-oxidation in GDM and T2DM [31,32].

Lipid peroxides are produced by polyunsaturated fatty acids (PUFAs) oxidative degradation, which generates products that exacerbate OS in tissues and cells. The 
A

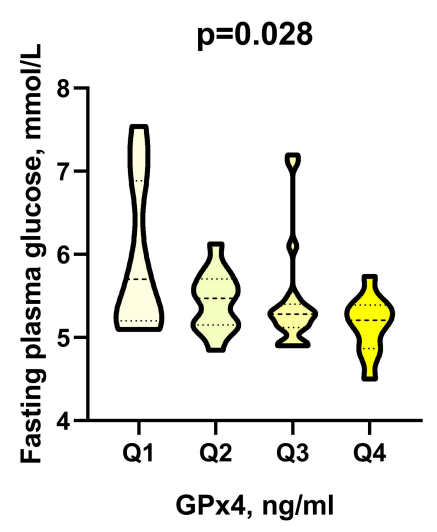

D

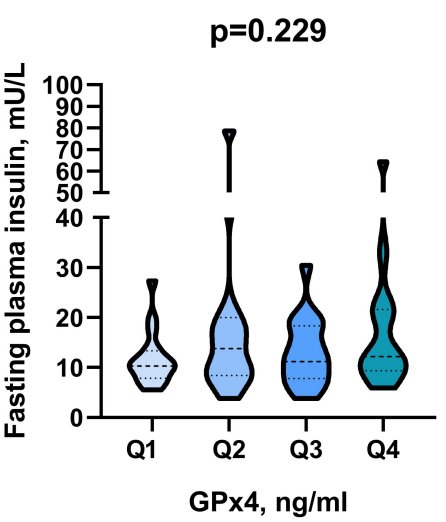

B

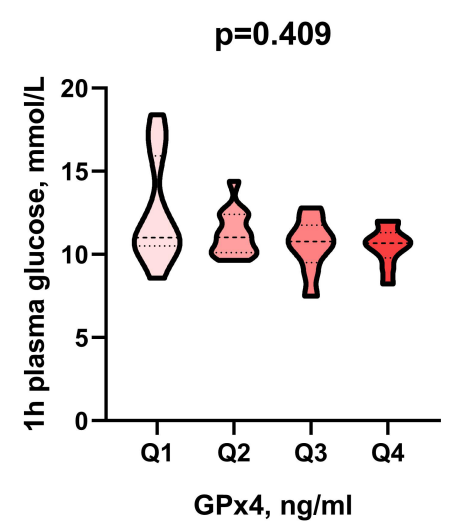

E

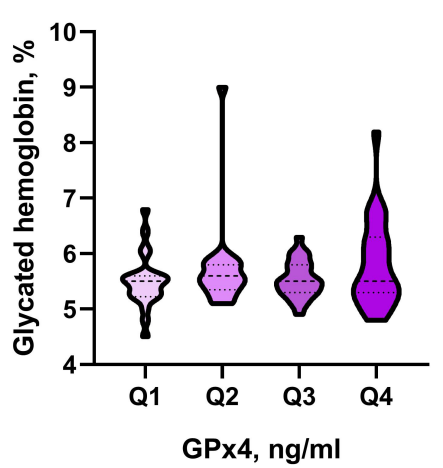

C

$\mathrm{p}=\mathbf{0 . 0 1 0}$

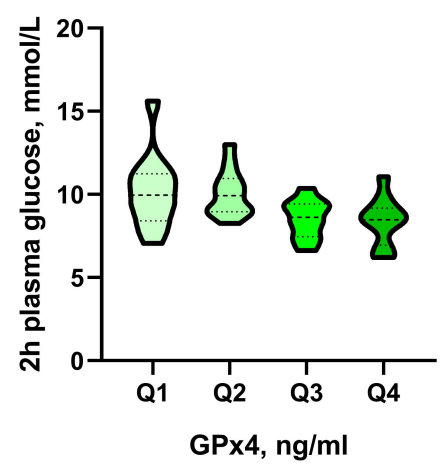

$\mathrm{F}$

$p=0.004$

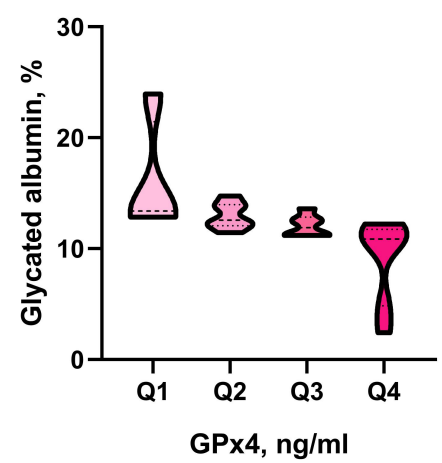

Fig. 4. The relationship between GPx4 and fasting plasma glucose (A), 1h-PG (B), 2 h-PG (C), fasting plasma insulin (D), glycated hemoglobin (E), and glycated albumin (F).

markers of lipid peroxidation include MDA and 8-isoprostaglandin $\mathrm{F} 2 \alpha$ (8-iso-PGF2 $\alpha$ ), whereas the antioxidant markers include catalase, GPx, and superoxide dismutase. The correlation between expression levels of the oxidative stress index, for example, MDA and 8-iso-PGF2 $\alpha$, in placental tissues or plasma of pregnant women with GDM was investigated [33-35]. In a previous study, Shang et al. [36] suggested that serum LPO markedly increased among GDM cases compared with normal pregnant women, and that LPO in the maternal plasma displayed a significant positive relationship with hemoglobin Alc values. As far as we are aware, the present study is the first to assess the relationship of LPO/GPx4 concentrations at 24-28 gestational weeks with glucose metabolism in the progression of pregnancy among GDM cases from the Chinese population. We found that GDM patients had higher LPO levels than normal controls $(p<0.001)$. Based on these findings, GDM cases had increased LPO concentrations, which were strongly associated with metabolic abnormalities among the gestational population and might serve as a predictor for metabolic abnormalities. By feeding Wistar rats a diet that contained $60 \%$ fructose (HFD) for 2,4 , or
6 weeks, Kitagama and colleagues discovered that the FFA concentration increased in a week-dependent manner. Additionally, the authors analyzed the role of HFD-mediated metabolic syndrome (MetS) in the rat tissue LPO concentration [37]. Four- and 6-week HFD feeding induced MetS with an increased serum LPO concentration.

Once gestational women were classified as NGT or GDM based on the blood glucose status, the present study showed that the GPx4 concentration was markedly decreased in the GDM group compared with the NGT group. Moreover, the GPx4 concentrations among gestational women displayed a linear correlation with the FPG, 2h$\mathrm{PG}$, and GA\% for glucose metabolism in Chinese pregnant women, even when adjusted for gestational age and age. In recent years, Krümmel and colleagues discovered markedly elevated GPx 4 concentrations within $\beta$-cells compared with the markedly decreased concentrations within additional islet cells associated with the $\beta$-cell susceptibility to toxic LPO accumulation [38]. Overall, the above results indicate the critical role of GPx4 in the function of $\beta$-cells under physiological conditions. Additionally, this study suggested that ferroptosis, another cell death form of $\beta$-cells, 

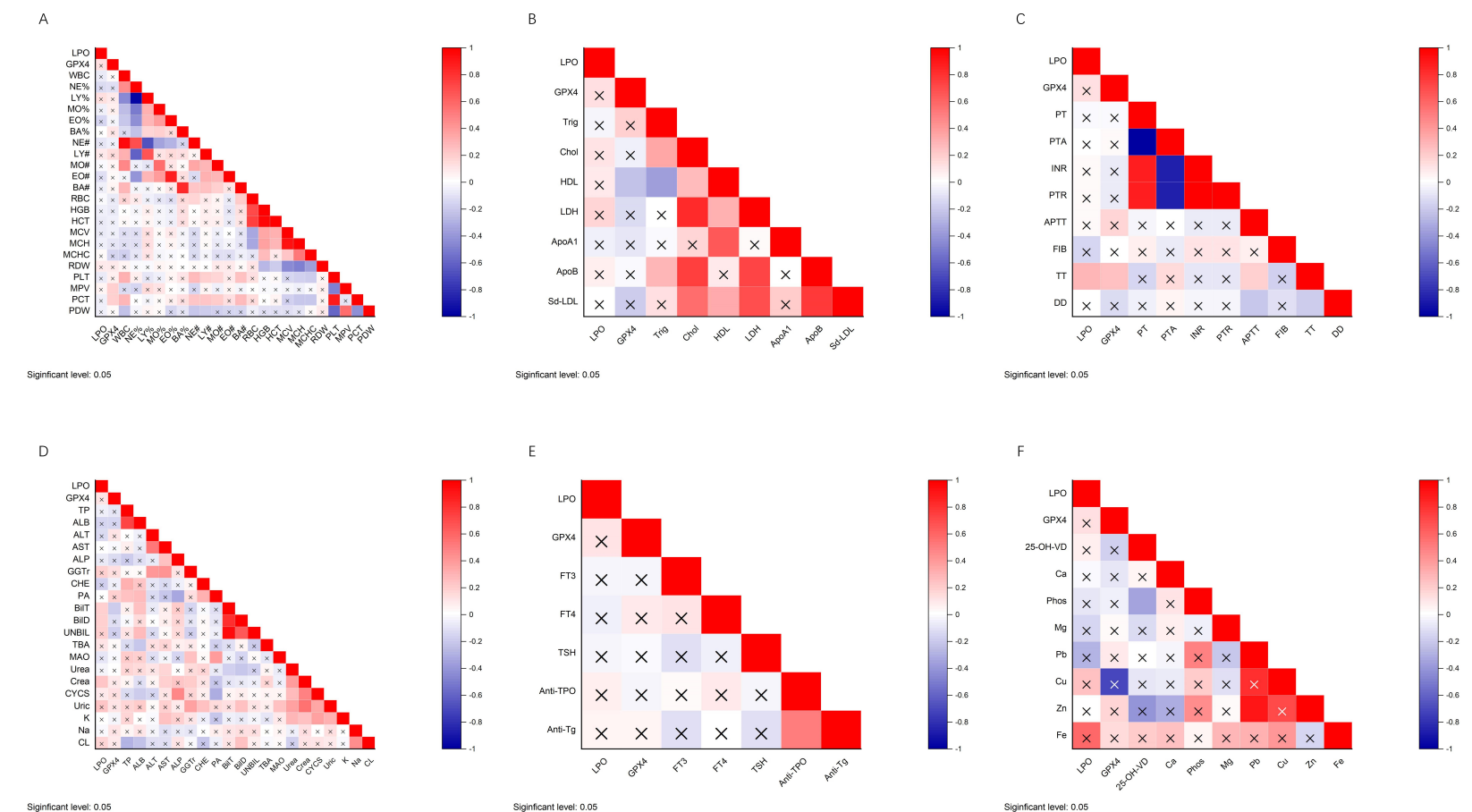

Fig. 5. The correlations between the LPO and GPx4 levels and the clinical features of the blood routine (A), serum lipid profile (B), blood coagulation function (C), liver function (D), thyroid function (E), and serum electrolyte (F).

A

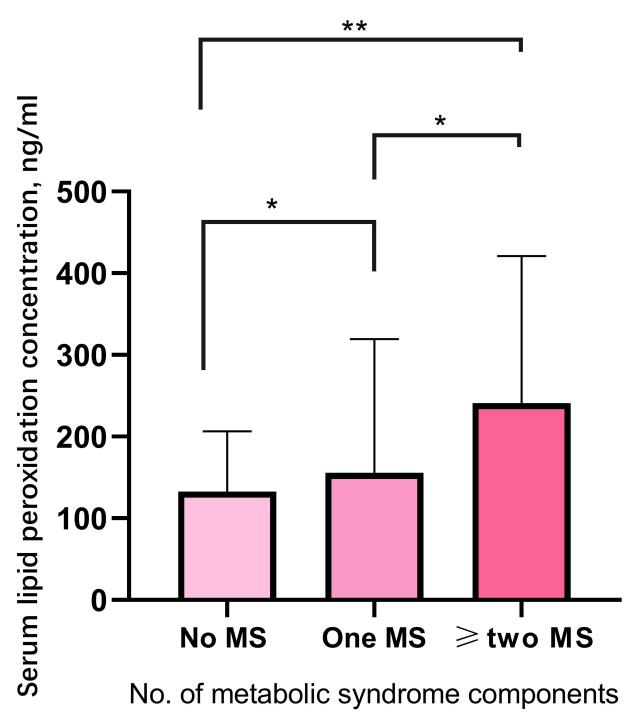

B

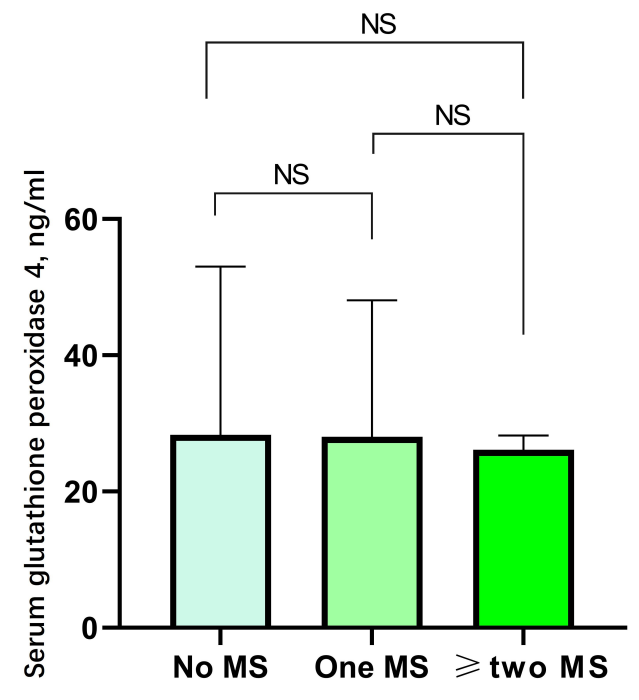

No. of metabolic syndrome components

Fig. 6. The serum LPO level (A) and GPx4 level (B) in the no metabolic abnormality, one metabolic abnormality, and two or more metabolic abnormalities groups.

did not involve the occurrence of a pro-inflammatory cytokine attack [38]. To date, no study has suggested that ferroptosis is involved in the T1DM pathogenic mechanism as the oxidative non-apoptotic cell death form. In addition, it remains unclear about the role of GPx4 as the main ferroptosis regulator and antioxidative enzyme.
The present study had some limitations. Firstly, there was a small sample size, which had some impact on the relationship of serum LPO and GPx4 concentrations with GDM occurrence. Secondly, this study determined LPO and GPx4 contents only in the second trimester. We did not perform dynamic monitoring of the of LPO or GPx4 concentrations or determine their clinical values in the third 
trimester. Thirdly, when collecting data, the time the LPO concentration was determined were inconsistent with that for the biochemical examination in some subjects (differences of up to 3 weeks), thus, potentially resulting in numerical differences and compromising the model interpretability.

\section{Conclusions}

In summary, the present study suggested that GPx4 played a vital role in glucose metabolism and that LPO was a possible risk factor associated with the metabolic abnormalities in the Chinese pregnant women. An increased serum LPO concentration might predict the incidence and degree of metabolic abnormalities among the Chinese gestational diabetes mellitus population in the future. To date, limited research has been conducted on the associations of serum GPx4 and LPO concentrations with GDM, such that more evidence-based studies are warranted. The small sample size and restricted geographical distribution were potential limitations in the present study. Consequently, a multiarea and multi-center prospective study with a large sample size should be conducted to illustrate the associations of serum LPO/GPx4 with GDM.

\section{Abbreviations}

BMI, body mass index; SBP, systolic blood pressure; DBP, diastolic blood pressure; TP, total protein; ALT, alanine aminotransferase; AST, aspartate aminotransferase; ALP, alkaline phosphatase; ALB, albumin; GGT, gammaglutamyl transpeptidas; PA1, pre-albumin; BilT, total bilirubin; $\mathrm{CB}$, conjugated bilirubin; $\mathrm{UCB}$, un-conjugated bilirubin; TBA, total bile acid; FPG, fasting plasma glucose; TG, triglyceride $\mathrm{TC}$, total cholesterol; HDL-C, high-density lipoprotein-cholesterol; LDL-C, low-density lipoprotein-cholesterol; ApoA1, apolipoprotein A1; AopB, apolipoprotein B. WBC, white blood cells; RBC, red blood cells; HGB, hemoglobin; PLT, platelet; BA, basophil; EO, eosinophil; LY, lymphocyte; MO, monocyte; NE, neutrophil; $\mathrm{MCH}$, mean corpuscular hemoglobin; $\mathrm{MCHC}$, mean corpuscular hemoglobin concentration; $\mathrm{MCV}$, mean corpuscular Volume; MPV, mean platelet volume; HCT, hematocrit; PCT, platelet hematocrit; PDW, platelet distribution width; RDW, red blood cell volume distribution width; APTT, activated partial thromboplastin time; PT, prothrombin time; PTA, prothrombin activity; INR, international normalized ratio; CRP, C-reactive protein; PSM, propensity score matching.

\section{Author contributions}

BZ and LS was responsible for the study design, collection of blood sample, experiment implementation, study screening, data analysis and interpretation, statistics analysis, and drafting. LS, BZ, and TZ were in charge of research and drafting. SH, BZ, and LS were responsible for data collection and analysis. All authors made contributions to study design and interpretation, and further drafting. LS was the guarantor.

\section{Ethics approval and consent to participate}

The Medical Ethics Committee of Shengjing Hospital of China Medical University approved our study protocol (Ethics Approval No. 2017PS066K). This study was performed following the Declaration of Helsinki (revised in October 2013, Fortaleza, Brazil). Informed written consent was obtained from each participant before participating in this study.

\section{Acknowledgment}

We thank International Science Editing (http:/www.internationalscienceediting.com) for editing this manuscript.

\section{Funding}

This research received no external funding.

\section{Conflict of interest}

The authors declare no conflict of interest.

\section{Supplementary material}

Supplementary material associated with this article can be found, in the online version, at https://www.imrpre ss.com/journal/FBL/27/2/10.31083/j.fbl2702068.

\section{References}

[1] Macaulay S, Dunger DB, Norris SA. Gestational diabetes mellitus in Africa: a systematic review. PLoS ONE. 2014; 9: e97871.

[2] Li KT, Naik S, Alexander M, Mathad JS. Screening and diagnosis of gestational diabetes in India: a systematic review and meta-analysis. Acta Diabetologica. 2018; 55: 613-625.

[3] Gao C, Sun X, Lu L, Liu F, Yuan J. Prevalence of gestational diabetes mellitus in mainland China: a systematic review and meta-analysis. Journal of Diabetes Investigation. 2019; 10: 154 162.

[4] Prentki M, Nolan CJ. Islet beta cell failure in type 2 diabetes. Journal of Clinical Investigation 2006; 116: 1802-1812.

[5] Catalano PM. Trying to understand gestational diabetes. Diabetic Medicine. 2014; 31: 273-281.

[6] Retnakaran R, Hanley AJG, Raif N, Connelly PW, Sermer M, Zinman B. Reduced Adiponectin Concentration in Women with Gestational Diabetes. Diabetes Care. 2004; 27: 799-800.

[7] Catalano PM, Huston L, Amini SB, Kalhan SC. Longitudinal changes in glucose metabolism during pregnancy in obese women with normal glucose tolerance and gestational diabetes mellitus. American Journal of Obstetrics and Gynecology. 1999; 180: 903-916.

[8] Clark CM, Qiu C, Amerman B, Porter B, Fineberg N, Aldasouqi $\mathrm{S}$, et al. Gestational Diabetes: should it be Added to the Syndrome of Insulin Resistance? Diabetes Care. 1997; 20: 867871.

[9] Fan J, Zhang T, Yu Y, Zhang B. Is serum zinc status related to gestational diabetes mellitus? A meta-analysis. Maternal \& Child Nutrition. 2021; 17: e13239. 
[10] Patrick TE, Hubel CA, Roberts JM. Evidence of increased oxidative stress, unexplained by lipid changes, is present in nulliparous black women from early gestation. Hypertension in Pregnancy. 2004; 23: 91-100.

[11] Sekeroğlu MR, Sahin H, Dülger H, Algün E. The effect of dietary treatment on erythrocyte lipid peroxidation, superoxide dismutase, glutathione peroxidase, and serum lipid peroxidation in patients with type 2 diabetes mellitus. Clinical Biochemistry. 2000; 33: 669-674.

[12] Karacay O, Sepici-Dincel A, Karcaaltincaba D, Sahin D, Yalvaç $\mathrm{S}$, Akyol M, et al. A quantitative evaluation of total antioxidant status and oxidative stress markers in preeclampsia and gestational diabetic patients in 24-36 weeks of gestation. Diabetes Research and Clinical Practice. 2010; 89: 231-238.

[13] Valko M, Jomova K, Rhodes CJ, Kuča K, Musílek K. Redoxand non-redox-metal-induced formation of free radicals and their role in human disease. Archives of Toxicology. 2016; 90 : 1-37.

[14] Valko M, Morris H, Cronin M. Metals, Toxicity and Oxidative Stress. Current Medicinal Chemistry. 2005; 12: 1161-1208.

[15] Ursini F, Maiorino M. Lipid peroxidation and ferroptosis: the role of GSH and GPx4. Free Radical Biology and Medicine. 2020; 152: 175-185.

[16] López-Tinoco C, Roca M, García-Valero A, Murri M, Tinahones FJ, Segundo C, et al. Oxidative stress and antioxidant status in patients with late-onset gestational diabetes mellitus. Acta Diabetologica. 2013; 50: 201-208.

[17] Alberti KG, Zimmet PZ. Definition, diagnosis and classification of diabetes mellitus and its complications. Part 1: diagnosis and classification of diabetes mellitus provisional report of a who consultation. Diabetic Medicine. 1998; 15: 539-553.

[18] Metzger BE, Gabbe SG, Persson B, Buchanan TA, Catalano PA, Damm $\mathrm{P}$, et al. International association of diabetes and pregnancy study groups recommendations on the diagnosis and classification of hyperglycemia in pregnancy. Diabetes Care. 2010; 33: 676-682.

[19] Niu J, Lei Q, Lü L, Wen J, Lin X, Duan D, et al. Evaluation of the diagnostic criteria of gestational metabolic syndrome and analysis of the risk factors. Zhonghua Fu Chan Ke Za Zhi. 2013; 48: 92-97. (In Chinese)

[20] Bertolini G, D’Amico R, Nardi D, Tinazzi A, Apolone G. One model, several results: the paradox of the Hosmer-Lemeshow goodness-of-fit test for the logistic regression model. Journal of Epidemiology and Biostatistics. 2000; 5: 251-253.

[21] Feng C, Jin Z, Chi X, Zhang B, Wang X, Sun L, et al. SHBG expression is correlated with PI3K/AKT pathway activity in a cellular model of human insulin resistance. Gynecological Endocrinology. 2018; 34: 567-573.

[22] Zhang B, Jin Z, Sun L, Zheng Y, Jiang J, Feng C, et al. Expression and correlation of sex hormone-binding globulin and insulin signal transduction and glucose transporter proteins in gestational diabetes mellitus placental tissue. Diabetes Research and Clinical Practice. 2016; 119: 106-117.

[23] Bandeira SDM, Guedes GDS, da Fonseca LJS, Pires AS, Gelain DP, Moreira JCF, et al. Characterization of blood oxidative stress in type 2 diabetes mellitus patients: increase in lipid peroxidation and SOD activity. Oxidative Medicine and Cellular Longevity. 2012; 2012: 819310.
[24] Ziegler D, Sohr CGH, Nourooz-Zadeh J. Oxidative stress and antioxidant defense in relation to the severity of diabetic polyneuropathy and cardiovascular autonomic neuropathy. Diabetes Care. 2004; 27: 2178-2183.

[25] Piwowar A, Knapik-Kordecka M, Warwas M. AOPP and its relations with selected markers of oxidative/antioxidative system in type 2 diabetes mellitus. Diabetes Research and Clinical Practice. 2007; 77: 188-192.

[26] Toescu V, Nuttall SL, Martin U, Kendall MJ, Dunne F. Oxidative stress and normal pregnancy. Clinical Endocrinology. 2002; 57: 609-613.

[27] McKinney ET, Shouri R, Hunt RS, Ahokas RA, Sibai BM. Plasma, urinary, and salivary 8-epi-prostaglandin f2alpha levels in normotensive and preeclamptic pregnancies. American Journal of Obstetrics and Gynecology. 2000; 183: 874-877.

[28] Arikan S, Konukoğlu D, Arikan C, Akçay T, Davas I. Lipid peroxidation and antioxidant status in maternal and cord blood. Gynecologic and Obstetric Investigation. 2001; 51: 145-149.

[29] Orhan H, Onderoglu L, Yücel A, Sahin G. Circulating biomarkers of oxidative stress in complicated pregnancies. Archives of Gynecology and Obstetrics. 2003; 267: 189-195.

[30] Xiang AH, Peters RK, Trigo E, Kjos SL, Lee WP, Buchanan TA. Multiple metabolic defects during late pregnancy in women at high risk for type 2 diabetes. Diabetes. 1999; 48: 848-854.

[31] Chen X, Scholl TO. Oxidative stress: changes in pregnancy and with gestational diabetes mellitus. Current Diabetes Reports. 2005; 5: 282-288.

[32] Lappas M, Mittion A, Permezel M. In response to oxidative stress, the expression of inflammatory cytokines and antioxidant enzymes are impaired in placenta, but not adipose tissue, of women with gestational diabetes. Journal of Endocrinology. 2010; 204: 75-84.

[33] Li H, Dong A, Lv X. Advanced glycation end products and adipocytokines and oxidative stress in placental tissues of pregnant women with gestational diabetes mellitus. Experimental and Therapeutic Medicine. 2019; 18: 685-691.

[34] Shang M, Dong X, Hou L. Correlation of adipokines and markers of oxidative stress in women with gestational diabetes mellitus and their newborns. Journal of Obstetrics and Gynaecology Research. 2018; 44: 637-646.

[35] Lappas M, Permezel M, Rice GE. Release of proinflammatory cytokines and 8-isoprostane from placenta, adipose tissue, and skeletal muscle from normal pregnant women and women with gestational diabetes mellitus. The Journal of Clinical Endocrinology and Metabolism. 2004; 89: 5627-5633.

[36] Shang M, Zhao J, Yang L, Lin L. Oxidative stress and antioxidant status in women with gestational diabetes mellitus diagnosed by IADPSG criteria. Diabetes Research and Clinical Practice. 2015 ; 109: 404-410.

[37] Kitagawa A, Ohta Y, Ohashi K, Yashiro K, Fukuzawa K. Effect of High Fructose-Induced Metabolic Syndrome on Tissue Vitamin E and Lipid Peroxide Levels in Rats. Journal of Nutritional Science and Vitaminology. 2020; 66: 200-206.

[38] Krümmel B, Plötz T, Jörns A, Lenzen S, Mehmeti I. The central role of glutathione peroxidase 4 in the regulation of ferroptosis and its implications for pro-inflammatory cytokine-mediated beta-cell death. Biochimica et Biophysica Acta (BBA) - Molecular Basis of Disease. 2021; 1867: 166114. 